Preprints of the

Max Planck Institute for

Research on Collective Goods

Bonn 2010/26

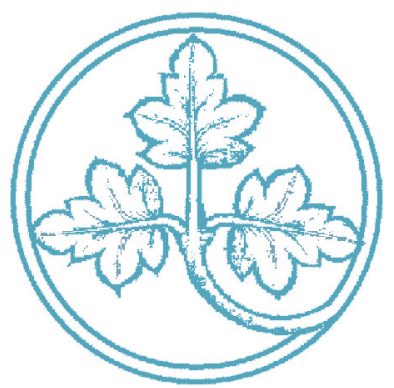

Skill Formation under

Incomplete Information

Philipp Weinschenk

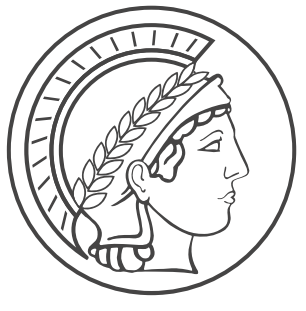

MAX PLANCK SOCIETY 


\section{Skill Formation under Incomplete Information}

Philipp Weinschenk

June 2010

Max Planck Institute for Research on Collective Goods, Kurt-Schumacher-Str. 10, D-53113 Bonn http://www.coll.mpg.de 


\title{
SKILL FORMATION UNDER INCOMPLETE INFORMATION
}

\author{
Philipp Weinschenk* \\ Bonn Graduate School of Economics \\ and Max Planck Institute for Research on Collective Goods
}

June 8, 2010

\begin{abstract}
We consider an economic model of child development with multiple stages. Due to incomplete information, parents are not able to tailor their investments to their child's type when the child is young. We show that incomplete information weakens the importance of early investments in children when inter-stage investments are easily substitutable, but strengthens them when substitution is difficult. The latter case is empirically relevant.

Keywords: Skill Formation, Education, Incomplete Information.

JEL-classification: I21, J13, D80.
\end{abstract}

\section{INTRODUCTION}

The formation of human capital is a central issue in economics. Cunha and Heckman (2007; henceforth CH) consider an economic model of child development, where the formation of human capital occurs in multiple stages via investments. They solve for the optimal intertemporal investment plan, which has important policy implications.

We extend their framework by assuming that children are differentiated in the sense that a child's type determines what type of investment

* Max Planck Institute for Research on Collective Goods, Kurt-Schumacher-Str. 10, 53113 Bonn, Germany, weinschenk@coll.mpg.de. I thank Cecile Aubert, Felix Bierbrauer, Stefanie Brilon, Stephan Dickert, Christoph Engel, Martin Hellwig, Michael Kurschilgen, and Andrea Wittke for helpful comments and suggestions. 
is most productive for him/her, and that this information is not available when a child is young. That is, there is incomplete information when the child is young. However, we assume that when a child is older its type is revealed. How does the optimal investment plan change as a result of incomplete information? Put differently, how should parents react to uncertainty about how to treat their young child best?

There are two intuitive guesses: (i) invest less in the early and more in the late stage, because late-stage investments are more efficient since they can be tailored to the child's type which is then known; (ii) invest more in the early and less in the late stage, because this guarantees that the effective investment in the early stage is not too bad.

We show that the answer is remarkably simple. It crucially depends on the substitutability of investment between stages. When investments are easily substitutable (easier than Cobb-Douglas), intuition (i) is right; when substitution is difficult (more difficult than Cobb-Douglas), (ii) is right. More specifically, incomplete information weakens the importance of early investments in children when inter-stage investments are easily substitutable, but strengthens them when substitution is difficult. The findings of Cunha, Heckman, and Schennach (forthcoming) indicate that the latter case is empirically relevant; see the discussion in Section 3.

In the next section, we first present CH's model. Then we extend it by introducing differentiated investments and incomplete information. In Section 3, we conclude and discuss the results.

\section{MODELS}

\subsection{CUNHA AND HECKMAN'S MODEL}

There is a child with two stages of childhood, $t=1,2$. A child's adult stock of skill $h$, also called human capital, is given by

$$
h=m\left(h^{p}, \theta_{1}, I_{1}, I_{2}\right),
$$

where $h^{p}$ is the skill of the parents, $\theta_{1}$ the child's initial ability, and $I_{1}$ and $I_{2}$ are investments in the early and the late stage, respectively. ${ }^{1}$ For

\footnotetext{
${ }^{1}$ We use a slightly different notation than $\mathrm{CH}$.
} 
concreteness $\mathrm{CH}$ consider the following form, where $I$ is given by a CES function:

$$
h=m\left(h^{p}, \theta_{1}, I\right), I=\left[\gamma I_{1}^{\phi}+(1-\gamma) I_{2}^{\phi}\right]^{1 / \phi} .
$$

The parameter $\gamma, 0<\gamma<1$, is interpreted as a skill multiplier. It influences the productivity of early investment not only in directly boosting $h$, but also in raising the productivity of $I_{2}$ by increasing the late-stage ability through high early-stage investments; see $\mathrm{CH}$ (p. 38). The parameter $\phi, \phi \leq 1$, describes how easily investments at different stages can be substituted for each other. For $\phi=1$, we have a linear relationship: $I=\left[\gamma I_{1}+(1-\gamma) I_{2}\right]$. That is, investments are perfect substitutes. For $\phi \rightarrow-\infty$, investments are not substitutable; the function is of the Leontief type. For $\phi=0$, one gets the Cobb-Douglas function. The elasticity of substitution is $1 /(1-\phi)$.

We assume that parents at the beginning of $t=1$ maximize the present value of the net wealth of their children

$$
\mathbb{E}[\pi]=w \mathbb{E}[h]-I_{1}-\frac{1}{1+r} I_{2}
$$

over $\left\{I_{1}, I_{2}\right\} .^{2}$ The costs of late-stage investments are discounted by the factor $1 /(1+r)$, where $r$ is the interest rate. The life-time discounted wage per unit of skill is denoted by $w$. So that an optimum exists, we assume that $d^{2} m(\cdot) / d I^{2}<0$. To guarantee that it is optimal to invest some positive amount, we assume that $\lim _{I \rightarrow 0} d m(\cdot) / d I$ is "sufficiently large". For $\phi<1$, optimization yields that the ratio of early-to-late investment is ${ }^{3}$

$$
\frac{I_{1}}{I_{2}}=\left(\frac{\gamma}{(1-\gamma)(1+r)}\right)^{\frac{1}{1-\phi}}
$$

$\mathrm{CH}$ interpret their formula as follows: "High productivity of initial investment (the skill multiplier $\gamma$ ) drives the parent toward making early investments. The interest rate drives the parent to invest late" (p. 39).

${ }^{2}$ Cunha, Heckman, Lochner, and Masterov (2005) also look at this maximization problem. An alternative approach is to consider a dynamic overlapping generations model (see the same paper or $\mathrm{CH}$ ). But, as the authors note, the main conclusions do not depend on which approach is used.

${ }^{3}$ For $\phi=1$ one gets corner solutions and the ratio need not be defined. 


\subsection{THE MODEL WITH INCOMPLETE INFORMATION}

We now extend the model by considering incomplete information. We assume that there are two types of investments at every stage: $\hat{I}_{t}$ and $\check{I}_{t}{ }^{4}$ For example, $\hat{I}_{t}$ may be the investment in child's athletic abilities whereas $\check{I}_{t}$ may denote investment in child's creativity. Both $\hat{I}_{t}$ and $\check{I}_{t}$ are measured in nominal units and are therefore called nominal investments. How the nominal investments combine to determine the effective investment $^{5}$ depends on the child's type $\alpha \in\{-\beta, \beta\}$ :

$$
I_{t}^{\text {effective }}=(1+\alpha) \hat{I}_{t}+(1-\alpha) \check{I}_{t} .
$$

With equal probability the child's type is $\beta$ or $-\beta$, where $0<\beta<1$. Hence, when the child is of type $\beta$, it is most productive to invest in $\hat{I}_{t}$ and not in $\check{I}_{t}$; it is the other way round when the type is $-\beta$. The size of the parameter $\beta$ captures how strongly the productivity of the intra-stage investments differs.

In $t=1$, the child's type $\alpha$ is not known to the parents. There is incomplete information. Hence they cannot be sure how best to tailor the investment to the child. ${ }^{6}$ Put differently, parents do not know whether they should invest in the child's athletic or creative abilities. In $t=2$, when the child is older, the parents learn the child's type.

As in CH's model, we assume that the relationship between the effective per stage investments and the effective investment $I$ is given by a CES function:

$$
I=\left[\gamma\left(I_{1}^{\text {effective }}\right)^{\phi}+(1-\gamma)\left(I_{2}^{\text {effective }}\right)^{\phi}\right]^{1 / \phi} .
$$

The parents' investment policy maximizes the present value of the net wealth of their children, that is, the present value of the child's skill minus

${ }^{4} \mathrm{CH}$ (and several other papers in which these authors are involved) also consider differentiated investments. But they do not consider incomplete information.

${ }^{5}$ Effective investments can be interpreted as productivity-adjusted nominal investments.

${ }^{6}$ We do not consider mechanisms that reveal the child's type. This is justified because young children are simply unable to reveal their types (or maybe they cannot be convinced to participate in any kind of mechanism). 
all nominal investments:

$$
\mathbb{E}_{\alpha}[\pi]=w \mathbb{E}_{\alpha}[h]-I_{1}^{T}-\frac{1}{1+r} I_{2}^{T},
$$

where we have defined the total nominal investment in stage $t$ as $I_{t}^{T}:=$ $\hat{I}_{t}+\check{I}_{t}$.

When parents invest in $t=2$, they know the child's type. Hence, it is optimal not to invest in the less productive investment: $\hat{I}_{2}=0$ when $\alpha=-\beta$ and $\check{I}_{2}=0$ when $\alpha=\beta$. We denote the highly productive investments by $I_{2}^{H}$. That is, $I_{2}^{H}=\hat{I}_{2}$ when $\alpha=\beta$ and $I_{2}^{H}=\check{I}_{2}$ when $\alpha=-\beta$.

L E M M A 1: It is optimal to choose $\hat{I}_{1}=\check{I}_{1}$ and $\left.I_{2}^{H}\right|_{\alpha=-\beta}=\left.I_{2}^{H}\right|_{\alpha=\beta}$. For $\phi<1$ it is optimal to invest in both stages.

Proof: See Appendix.

The first part of Lemma 1 says that it is optimal to diversify investments completely by choosing $\hat{I}_{1}=\check{I}_{1} \cdot{ }^{7}$ The second part states that although the type of the late-stage investment depends on $\alpha$, the size of the late-stage investment, i.e., $I_{2}^{H}$, is independent of $\alpha$.

As in CH's model, we would like to determine the optimal ratio of early-to-late total nominal investments. From the first-order conditions of the problem (see the Appendix) and Lemma 1, one directly gets that for $\phi<1$ the ratio is

$$
\frac{I_{1}^{T}}{I_{2}^{T}}=\left(\frac{\gamma}{(1-\gamma)(1+r)(1+\beta)^{\phi}}\right)^{\frac{1}{1-\phi}} .
$$

P R O P O I T I O N 1: Suppose that $\phi<1$. When $\phi>0$, the ratio of early-to-late total nominal investments with incomplete information is smaller than without incomplete information. When $\phi<0$, the ratio is larger with incomplete information.

The intuition is as follows. When investments can be substituted easily (easier than Cobb-Douglas), a low early-stage effective investment

\footnotetext{
${ }^{7}$ Completely diversify means that parents invest the same amount in, e.g., the child's athletic and creative abilities.
} 
can easily be compensated by a high late-stage investment. Late-stage investments have the advantage that they can be tailored to the child's type. Hence, it is optimal to invest little in the early and much in the late stage. However, when investments are difficult to substitute (more difficult than Cobb-Douglas), this is not the case. A low early-stage effective investment can only be compensated by a very high late-stage investment. This would be very costly. Hence, it is optimal to invest much in the early stage to make sure that the effective early-stage investment is substantial. $^{8}$

Should the parents invest more in the early than in the late stage of childhood? Looking at (2) and (6) yields the following answer. ${ }^{9}$

P R O P O S I T O N 2: The early-stage nominal investments exceed the late-stage investments in the model without incomplete information if $\gamma>(1-\gamma)(1+r)$. With incomplete information this is true for $\gamma>$ $(1-\gamma)(1+r)(1+\beta)^{\phi}$. It is the other way round when the formulas hold with $<$.

So when $\phi>0$, i.e., when substitution is easier than with a CobbDouglas function, the skill multiplier $\gamma$ must be larger in the model with incomplete information than in the model without so that the earlystage investment exceeds the late-stage investment. For $\phi<0$, i.e., when substitution is more difficult than with a Cobb-Douglas function, the multiplier $\gamma$ can be lower.

To sum up both propositions, incomplete information weakens the importance of early investments in children when inter-stage investments are easily substitutable. When substitution is difficult, early investments

${ }^{8}$ The results we found are mathematically closely related to Acemoglu (2002). He considers how the augmentation of one factor changes the relative marginal products of both factors of production. He shows that when the elasticity of substitution is above 1 , then the relative marginal product of the factor which is augmented improves. When the elasticity of substitution is below 1 , then it is the other way round. In our model, incomplete information augments investments in the late stage relative to investments in the early stage.

${ }^{9}$ As in CH's model, the investment ratio need not be defined for $\phi=1$. It is easily shown that the result stated in Proposition 2 is also valid for $\phi=1$. 
become more important.

For completeness, consider the case with differentiated investments, but without incomplete information. Then it is optimal to invest only in the productive type of investment at both stages. The parameter $\beta$ appears in both first-order conditions in the same way. Hence, the term $\beta$ cancels out in the investment ratio and the ratio is as in CH's model.

\section{CONCLUSIONS AND DICUSSION}

We have extended the model of $\mathrm{CH}$ by introducing incomplete information about a child's type when it is young. We have shown that incomplete information weakens the importance of early investment in children when inter-stage investments are easily substitutable, but strengthens their importance when substitution is difficult.

Cunha, Heckman, and Schennach (forthcoming) estimate elasticities of substitution in a model which does not consider incomplete information and differs from our setting in other aspects also. Therefore, their estimates cannot be fully transfered to our model. The authors show that the substitution in the late stage of childhood is relatively difficult (more difficult than Cobb-Douglas). This holds for cognitive as well as noncognitive skills and for several specifications. Therefore, these findings indicate that incomplete information makes early investments in children more important. Therefore, we strengthen CH's result that early investments are of utmost importance.

We have assumed that the differentiated investments at a stage combine in a linear way to the effective investment, see (3). This simplification can be defended as follows: with a more complicated functional form, it stays true that knowing a child's type allows tailored investments. Hence, investing later yields a return on the effective late-stage investment which is greater, say by a factor $(1+\beta)$, than the expected return early investments have on the effective early-stage investment. Therefore, the linear specification is a reduced form of the more general specification. These arguments show that the inter-stage investment problem does not change due to a more general specification. However, the intrastage investment problem changes. With a more general specification it 
may be optimal to invest in the late stage of childhood in a child's ability in which he/she is less talented.

Eliciting the incomplete information through scientific tests allows a tailored investment policy also for young children. Then, the same adult skill levels are attainable with lower investments. Alternatively, with the same investments, higher skill levels can be achieved. Hence, those tests are important to improve adult skill and the effectiveness of investments.

The model we consider can be interpreted more broadly. There is a multi-stage investment problem with only initial uncertainty about the most productive way to invest. An example may be the problem of global warming, where it is not yet clear how carbon dioxide (or other climate gases) can technically most efficiently be deterred.

\section{APPENDIX: PROOF OF LEMMA 1}

The following properties of the CES function, as specified in (1), are useful:

(i) $\frac{d I}{d I_{t}}$ is positive and homogenous of degree 0 .

(ii) $\frac{d^{2} I}{d I_{1} d I_{2}}$ is positive for $\phi<1$ and zero for $\phi=1$.

(iii) $\frac{d^{2} I}{d I_{t}^{2}}$ is negative for $\phi<1$ and zero for $\phi=1$.

(iv) For $\phi<1$ and $I_{s}>0, \lim _{I_{t} \rightarrow 0} \frac{d I}{d I_{t}}=\infty$, where $s \neq t$.

The first-order conditions of (5) are:

$$
\begin{aligned}
\frac{d \pi}{d I_{2}^{H}} & =\left.w \frac{d m(\cdot)}{d I}\right|_{\alpha}[\cdot]_{\alpha}^{1 / \phi-1}(1-\gamma)\left(\left.(1+\beta) I_{2}^{H}\right|_{\alpha}\right)^{\phi-1}(1+\beta)-\frac{1}{1+r}=0 ; \\
\frac{d \mathbb{E}_{\alpha}[\pi]}{d \hat{I}_{1}} & =\left.\frac{1}{2} w \frac{d m(\cdot)}{d I}\right|_{\alpha=\beta}[\cdot]_{\alpha=\beta}^{1 / \phi-1} \gamma\left((1+\beta) \hat{I}_{1}+(1-\beta) \check{I}_{1}\right)^{\phi-1}(1+\beta) \\
& +\left.\frac{1}{2} w \frac{d m(\cdot)}{d I}\right|_{\alpha=-\beta}[\cdot]_{\alpha=-\beta}^{1 / \phi-1} \gamma\left((1-\beta) \hat{I}_{1}+(1+\beta) \check{I}_{1}\right)^{\phi-1}(1-\beta) \\
& -1=0 ; \\
\frac{d \mathbb{E}_{\alpha}[\pi]}{d \check{I}_{1}} & =\left.\frac{1}{2} w \frac{d m(\cdot)}{d I}\right|_{\alpha=\beta}[\cdot]_{\alpha=\beta}^{1 / \phi-1} \gamma\left((1+\beta) \hat{I}_{1}+(1-\beta) \check{I}_{1}\right)^{\phi-1}(1-\beta) \\
& +\left.\frac{1}{2} w \frac{d m(\cdot)}{d I}\right|_{\alpha=-\beta}[\cdot]_{\alpha=-\beta}^{1 / \phi-1} \gamma\left((1-\beta) \hat{I}_{1}+(1+\beta) \check{I}_{1}\right)^{\phi-1}(1+\beta) \\
& -1=0 .
\end{aligned}
$$


$[\cdot]_{\alpha}$ is the square bracket of (4) evaluated at $\alpha$. For the first-order conditions of $\hat{I}_{1}$ and $\breve{I}_{1}$ we have used the Envelope theorem. Note that we cannot be sure that in the optimum the first-order conditions must be satisfied.

Due to the assumptions on $m(\cdot)$ it cannot be optimal not to invest at all. Additionally, due to the concavity of $m(\cdot)$ an optimum exists.

Part 1: It holds that $\hat{I}_{1}=\check{I}_{1}$.

The case $\phi<1$. Property (iv) of the CES function implies that it is optimal to invest a positive amount in both stages. Which proves the last part of Lemma 1 and implies that the first-order condition of $I_{2}^{H}$ must be fulfilled in the optimum. Since $\frac{d^{2} I}{d I_{t}^{2}}<0$ for $\phi<1$ and $d^{2} m(\cdot) / d I^{2}<0$ the optimal $I_{2}^{H}$ is unique. Moreover, since $\frac{d I}{d I_{t}}$ is homogenous of degree 0 and $d^{2} m(\cdot) / d I^{2}<0$ in the optimum $\frac{I_{2}^{H}}{I_{1}^{\text {effective }}}$ is decreasing in $I_{1}^{\text {effective }}$.

Suppose that $\hat{I}_{1}<\check{I}_{1}$. Then $\frac{d \mathbb{E}_{\alpha}[\pi]}{d \hat{I}_{1}} \leq \frac{d \mathbb{E}_{\alpha}[\pi]}{d \check{I}_{1}}$. Hence,

$$
\begin{gathered}
\left.\frac{d m(\cdot)}{d I}\right|_{\alpha=\beta}[\cdot]_{\alpha=\beta}^{1 / \phi-1}\left((1+\beta) \hat{I}_{1}+(1-\beta) \check{I}_{1}\right)^{\phi-1} \\
\leq\left.\frac{d m(\cdot)}{d I}\right|_{\alpha=-\beta}[\cdot]_{\alpha=-\beta}^{1 / \phi-1}\left((1-\beta) \hat{I}_{1}+(1+\beta) \check{I}_{1}\right)^{\phi-1} .
\end{gathered}
$$

From before we know that the first-order condition of $I_{2}^{H}$ must be fulfilled in the optimum. Inserting it into the previous inequality yields

$$
\left(\frac{\left.I_{2}^{H}\right|_{\alpha=\beta}}{\left.I_{1}^{\text {effective }}\right|_{\alpha=\beta}}\right)^{1-\phi} \leq\left(\frac{\left.I_{2}^{H}\right|_{\alpha=-\beta}}{\left.I_{1}^{\text {effective }}\right|_{\alpha=-\beta}}\right)^{1-\phi} \text {. }
$$

Due to $\hat{I}_{1}<\check{I}_{1}$ we have $\left.I_{1}^{\text {effective }}\right|_{\alpha=\beta}<\left.I_{1}^{\text {effective }}\right|_{\alpha=-\beta}$. Since in the optimum $\frac{I_{2}^{H}}{I_{1}^{\text {effective }}}$ is decreasing in $I_{1}^{\text {effective }}$, see before, (7) cannot be fulfilled. Also $\hat{I}_{1}>\check{I}_{1}$ yields a contradiction. Hence, $\hat{I}_{1}=\check{I}_{1}$.

The case $\phi=1$. With $\phi=1$ there are either corner solutions in which it is optimal to invest in only one stage, or there is an indifference. In the latter case, it is weakly optimal to choose $\hat{I}_{1}=\check{I}_{1}$. In the former case, it is either optimal (i) not to invest in the early stage, or (ii) it is optimal not to invest in the late stage. In case (i) $\hat{I}_{1}=\check{I}_{1}=0$. In case (ii) we 
must have $\hat{I}_{1}, \check{I}_{1}>0$ which implies $\frac{d \mathbb{E}_{\alpha}[\pi]}{d \hat{I}_{1}}=\frac{d \mathbb{E}_{\alpha}[\pi]}{d \check{I}_{1}}$. So

$$
\begin{aligned}
& \left.\frac{1}{2} \frac{d m(\cdot)}{d I}\right|_{\alpha=\beta}(1+\beta)+\left.\frac{1}{2} \frac{d m(\cdot)}{d I}\right|_{\alpha=-\beta}(1-\beta) \\
= & \left.\frac{1}{2} \frac{d m(\cdot)}{d I}\right|_{\alpha=\beta}(1-\beta)+\left.\frac{1}{2} \frac{d m(\cdot)}{d I}\right|_{\alpha=-\beta}(1+\beta)
\end{aligned}
$$

which simplifies to

$$
\left.\frac{d m(\cdot)}{d I}\right|_{\alpha=\beta}=\left.\frac{d m(\cdot)}{d I}\right|_{\alpha=-\beta} .
$$

Since $I_{2}^{H}=0$ this requires $\hat{I}_{1}=\check{I}_{1}$.

Part 2: It holds that $\left.I_{2}^{H}\right|_{\alpha=-\beta}=\left.I_{2}^{H}\right|_{\alpha=\beta}$.

The case $\phi<1$. From Part 1 we know that $\hat{I}_{1}=\check{I}_{1}>0$. This directly implies (see the first-order condition of $I_{2}^{H}$ ) that in the optimum, although the type of the late-stage investment depends on $\alpha$, the size of the late-stage investments $I_{2}^{H}$ is independent of $\alpha$.

The case $\phi=1$. When it is optimal to invest only in the late stage, the same arguments as with $\phi<1$ apply. When it is optimal only to invest in the early stage, we have $I_{2}^{H}=0$ for both, $\alpha=\beta$ and $\alpha=-\beta$. When there is a case of indifference, it is weakly optimal to choose $\hat{I}_{1}=\check{I}_{1}$, see Part 1. Then the the first-order condition of $I_{2}^{H}$ implies that $I_{2}^{H}$ is independent of $\alpha$.

\section{LITERATURE}

Acemoglu, Daron (2002). Directed Technical Change. Review of Economic Studies 69, 781-809.

Cunha, Flavio and James Heckman (2007). The Technology of Skill Formation. American Economic Review: Papers and Proceedings 97, 31-47.

Cunha, Flavio, James Heckman, Lance Lochner, and Dimitriy Masterov (2005). Interpreting the Evidence on Life Cycle Skill Formation. In Erik Hanushek and F. Welch (Ed.), Handbook of the Economics of Education 1, North Holland.

Cunha, Flavio, James Heckman, and Susanne Schennach (forthcoming). Estimating the Technology of Cognitive and Noncognitive Skill Formation. Econometrica. 